Reprod. Nutr. Dévelop., 1982, 22 (5), 753-766.

\title{
Déterminisme génétique de l'inversion du sexe femelle accompagnant le croisement de deux sous-espèces d'/dotea balthica (Pallas). I. - Résultats concernant les phénotypes albafusca, bilineata et uniformis
}

\author{
Emmanuelle LEGRAND-HAMELIN, J.-J. LEGRAND
}

avec la collaboration technique de C. LE BOTE et G. HEILY

Laboratoire de Physiologie et Génétique des Crustacés, E.R.A. CNRS n०230, 40, avenue du Recteur-Pineau, 86022 Poitiers Cedex.

Summary. Genetic determinism of the female sex inversion accompanying the crossing of two subspecies of Idotea balthica (Pallas). I. - Results on albafusca, bilineata and uniformis phenotypes.

A reversal of sexual phenotype resulting in the appearance of neo-males and neofemales occurs in the populations of two marine subspecies of /dotea balthica. Sex inversions are rare in $\%$ b. basteri but more frequent in $I . b$. tricuspidata. By crossing these two subspecies, the rate of neo-males $\left(t_{i}\right)$ was considerably increased, allowing us to study the determination of sex inversion.

The $/$. b. tricuspidata used in this study came from the offspring (27 p. 100 neo-males) of one gravid albafusca female $\left(A_{1}\right)$ collected in La Rochelle (LR); these offspring had been studied for five generations. The $/ . b$. basteri came from offspring of females collected in Marseille (M), Racou (eastern Pyrenees) and Tunis. The $\chi^{2}$ or Mann-Whitney test was used to compare the results of the different crosses.

The female /dotea balthica is heterogametic (WZ); the male is homogametic (ZZ). The loci of the genes which determine the colored phenotypes, albafusca (A) and bilineata (B), are situated on the $W$ heterochromosome. The fundamental phenotype, uniformis $(U)$, is the wild type, according to classical genetics. The males used were either $\mathrm{ZZ}$ and uniformis or WZ (neo-males) and albafusca.

1) Ten crosses: \& A basteri (M) $\times$ o U tricuspidata (LR) gave a variety of offsprings $\left(\chi^{2}=56-\right.$ d.f. $\left.=9\right)$, two having an $A_{1}$-like $t_{i}$ and the other eight no or very few neomales.

2) Backcrosses (from $C_{R F}$ to $C R F_{5}$ ) between hybrid females and $U$ La Rochelle males : in $\mathrm{CRF}_{2}$, the $t_{i}$ increased significantly and in $\mathrm{CRF}_{3}, \mathrm{CRF}_{4}$ and $C R F_{5}$ it reached the $t_{i}$ of the $A_{1}$ lineage.

3) In the $\& F_{1} \times$ or $U_{M}$ backcrosses, the $t_{i}$ was nearly zero, thus being comparable with the basteri lineage of Marseille.

4) When $\& A_{L R} \times O^{*} U_{M}$ of some lineages was crossed, the expressivity of the phenotype $A$ was reduced (from $A$ to $U$ ) only in neo-males. The penetrance of gene $A$ did not vary significantly in seven crosses from $F_{1}$ to $C R F_{2}$.

5) The crossing of $\mathrm{O}^{\circ} \mathrm{O}^{*} \mathrm{U}_{\mathrm{LR}}$ with $९ \subsetneq$ basteri $\mathrm{B}$ or $\mathrm{U}$ from various populations showed that only some $B$ and $U$ females were likely to favourize sex inversion in their daughters; the resulting $t_{i}$ was ten times higher than with $A$ females. 
These results led to the following conclusions :

- crosses 1, 2 and 3 showed that reinforcement of the tricuspidata genotype of La Rochelle, by means of a regular addition of mainly autosomal genes, induced an increase in the $t_{i}$, whereas reinforcement of the basteri genotype induced a decrease in that rate. The results supported the hypothesis of a polyfactorial determination of sex involving autosomal, or partly sex-linked, factors acting as inhibitors of the major female gene(s) :

- crosses 4 showed that the reduction of gene $A$ expressivity was always associated with the sex inversion of genetic hybrid females; that process also seemed to be controled by some autosomal or partly sex-linked recessive genes, different from those of the preceding complex and acting as inhibitors of the major female-determining factor(s) situated on the $W$ heterochromosome.

The existence of $Z Z$ neo-females (mentioned here) also demonstrated the presence of autosomal factor acting as inhibitors of the major male-determining factor(s).

\section{Introduction.}

L'espèce polytypique /dotea ba/thica compte notamment deux sous-espèces marines, l'une, I. b. tricuspidata, se trouve sur les côtes de l'Atlantique, de la Manche et de la mer du Nord, l'autre, I. b. basteri, se rencontre sur les côtes méditerranéennes. Des gènes de couleur strictement liés au chromosome $W$, en particulier albafusca et bilineata, qui n'occupent pas le même locus, ont permis à l'un de nous (Tinturier-Hamelin, 1963) d'apporter la preuve génétique de l'existence, chez cette espèce, d'un mécanisme hétérochromosomique de détermination sexuelle du type $q W Z$, ơ $Z Z$ qui a pu être confirmé par l'emploi de néomâles expérimentaux (Legrand-Hamelin, 1977).

On sait que chez cette espèce les gamètes des deux sexes offrent $n=29$ chromosomes (Artault, 1977) mais les hétérochromosomes n'ont pas été repérés cytologiquement. Si la représentation classique de chacun des deux sexes par une constitution héréro- ou homogamétique rend compte de l'égalité des sexes constatée dans la plupart des croisements, et de la transmission des caractères de coloration, elle est incapable de prévoir les phénomènes d'inversion spontanée du phénotype sexuel qui conduisent à la réalisation soit de néo-mâles, soit de néofemelles, soit de ces deux types d'inversés dans de nombreuses descendances. Une étude préliminaire de ces inversés a amené l'un d'entre nous (TinturierHamelin, 1963) à émettre I'hypothèse que l'inversion du sexe chez /dotea ba/thica offre un déterminisme génétique voisin de celui évoqué par Winge et Ditlevsen (1948) puis par Yamamoto (1959) chez les poissons cyprinodontiformes, à savoir que des gènes multiples autosomiques féminisants ou masculinisants peuvent avoir une action sur le sexe capable de supplanter celle des déterminants sexuels hétérochromosomiques. D'une façon générale, les inversés sont beaucoup moins nombreux dans les descendances $d^{\prime} l$. b. basteri que dans celles d'l. b. tricuspidata. Après avoir remarqué récemment que l'hybridation entre basteri et tricuspidata renforçait, dans certains cas, considérablement l'inversion des femelles en néo-mâles, il nous a paru intéressant de reprendre, par ce biais, le problème du déterminisme génétique de ce type d'inversion, et par là même de préciser le rôle des différents types de gènes impliqués dans la détermination du sexe chez /. ba/thica. 


\section{Matériel et méthodes.}

Rappelons que /. b., sous réserve de soins constants, s'élève facilement au laboratoire ; à $20^{\circ} \mathrm{C}$, elle devient apte à se reproduire 6 semaines après sa naissance et peut effectuer jusqu'à 6 pontes successives (une tous les 18 jours). Elle est donc favorable à une étude génétique.

Les $/$. b. tricuspidata des deux sexes utilisées dans les croisements proviennent exclusivement d'une lignée relativement riche en néo-mâles, issue d'une femelle albafusca $A_{1}$ trouvée gravide à La Rochelle (LR).

Les /. b. basteri utilisées proviennent soit de lignées issues de femelles récoltées gravides à Marseille (M), au lieu dit la Pointe Rouge, au Racou près d'Argelès-sur-Mer (Pyrénées Orientales) et à Tunis (Tunisie), soit d'individus prélevés directement dans les populations naturelles de ces localités.

Les caractéristiques de chacune des lignées ou populations utilisées seront données au début de chaque étude des croisements dans lesquelles elles interviennent. Chaque descendance sera représentée par un rapport $(R)=$ néo- $O^{*} O^{*} /$ O $\$$ de même phénotype coloré, à partir duquel peut être déduit le taux d'inversion : $t_{i}=\frac{\text { néo- } \sigma o ̛}{\text { néo- } \sigma^{\prime} \sigma^{\prime}+q Q} \times 100$. Le nombre des mâles uniformis (U) n'a été généralement rapporté que lorsqu'il s'est écarté significativement du nombre prévu par les équations qui illustrent les différents types de croisements, tel celui d'une femelle albafusca (A) par un mâle uniformis (U) :
a $\$$
(A) $W_{A} Z \times \sigma^{n}$
(U) (ZZ) $\rightarrow$ ơ
(U) $Z Z+W_{A} Z$ lo $q$
$A+$ néo-ơ ơ A)

Pour juger de l'homogénéité des rapports $\mathrm{R}$ concernant un même type de croisements, nous avons employé le test du $\chi^{2}$ 'au niveau 0,05 . Afin de tester l'effet d'un type de croisement sur le taux des néo-mâles, en cas d'ensembles hétérogènes, la comparaison des différents groupes de rapports $\mathrm{R}$ a été effectuée à l'aide du test non paramétrique de Mann-Whitney (1947) au niveau 0,05 (/n Faverge, 1962). L'ensemble des rapports $R$ est ainsi considéré comme un échantilIon des résultats caractérisant une lignée ou un type donné de croisements.

\section{Résultats.}

\section{I. - Croisements faisant intervenir le phénotype albafusca (A)}

1) Lignées utilisées.

La lignée tricuspidata $A_{\uparrow}$ a fourni, au fil des 5 générations étudiées, les rapports $R$ suivants concernant le phénotype $A: F_{1}: 7 / 16 ; F_{2}: 41 / 809 / 264 / 27$ $4 / 9 ; F_{3}: 8 / 289 / 19 ; F_{4}: 14 / 43 ; F_{5}: 4 / 22$. Cette lignée est homogène $\left(\chi^{2}=9,03-\right.$ d.l. $\left.=8\right)$; le $t_{i}$ moyen est de 27 p. 100 .

Les individus basteri utilisés proviennent de la descendance de plusieurs femelles A récoltées ovigères à Marseille et qui ont respectivement donné comme rapports $\mathrm{R}$ :

- en $F_{1}:$ \& $A_{1} 0 / 276$, \& $A_{2} 1 / 141$, \& $A_{3} 0 / 158, q A_{4} 0 / 111$, \& $A_{5} 0 / 89$, ㅇ $A_{6} 1 / 266, \& A_{7} 0 / 191$, \& $A_{8} 0 / 33$; les inversés sont donc exceptionnels. 
- dans les générations ultérieures de la $q A_{1}$, les rapports $R$ sont les suivants : $F_{2} 0 / 330 / 14 ; F_{3} 0 / 691 / 200 / 270 / 27 ; F_{4} 0 / 300 / 18 ; F_{5} 0 / 38$. Le $t_{i}$ demeure très faible, du même ordre que celui des descendances de $F_{1}$.

2) $F_{1}$ : \& A basteri $\times$ O U tricuspidata.

Dix croisements réalisés ont donné respectivement les rapports $R_{A}: 14 / 62$ $8 / 331 / 580 / 851 / 180 / 401 / 381 / 533 / 331 / 102$. Ces descendances constituent un ensemble très hétérogène $\left(\chi^{2}=56,09-\right.$ d.I. $\left.=9\right)$. Dans huit d'entre elles, le $t_{i}$, très faible ou nul, est très voisin de celui qui caractérise la population marseillaise employée ; seules, les deux premières offrent un $t_{i}$ non significativement différent de celui qui caractérise la souche rochellaise $\left(\chi^{2}\right.$ pour 1 d.I. $=0,89$ et 0,46 par rapport à la $F_{1}$ de la femeile (LR) $A_{1}$ ).

3) Croisement en retour: $Q F_{1} \times$ or $U$ tricuspidata.

Onze croisements de ce type, réalisés à partir des $\$$ de la $F_{1}$ caractérisée par $R_{A}=1 / 58$, ont donné les rapports $R_{A}: 9 / 55 \quad 10 / 100 \quad 28 / 98 \quad 17 / 81 \quad 13 / 85$ $13 / 112 \quad 19 / 133 \quad 7 / 97 \quad 18 / 148 \quad 18 / 87 \quad 2 / 26$. Cet ensemble est hétérogène $\left(\chi^{2}=20,05-\right.$ d.I. $\left.=10\right)$ avec un $t_{i}$ offrant une progression continue de 7 à 22 p. 100 de néo-mâles $A$; la comparaison de cet ensemble de $C R F_{2}$ et de la $F_{1}$ précédente, par le test de Mann-Whitney, montre un accroissement significatif du $t_{i}$. II $y a$, a fortiori, une différence très significative entre le taux bas d'inversion dans la $F_{1}$ d'origine $\left(R_{A}=1 / 58\right)$ et ceux qui caractérisent l'ensemble des $C R F_{2}$.

4) Croisements en retour ultérieurs: $\mathrm{CRF}_{3}, \mathrm{CRF}_{4}, \mathrm{CRF}_{5}$.

Le génotype tricuspidata a été renforcé par des croisements en retour successifs des femelles hybrides avec des mâles $U$ rochellais. Les rapports $R_{A}$ suivants ont été obtenus $-\mathrm{CRF}_{3}$ (à partir du $\mathrm{CRF}_{2}$ 18/87) : 20/67 6/28 3/4 14/94; $\mathrm{CRF}_{4}$ (à partir du $\mathrm{CRF}_{3}$ 14/94): 17/27 26/53, enfin $\mathrm{CRF}_{5}$ (issus du $\mathrm{CRF}_{4}$ 26/53) : $8 / 223 / 19$. Cet ensemble est hétérogène $\left(\chi^{2}=18,72-\right.$ d.I. $\left.=7\right)$. Sa comparaison à l'aide du test de Mann-Whitney avec l'ensemble des $\mathrm{CRF}_{2}$ montre un accroissement significatif $d u t_{i}$. La comparaison à l'aide du même test avec la ligne $A_{1}$ rochellaise ne révèle pas de différence significative dans le $t_{i}$, qui varie ici de 13,6 à 42,8 p. 100 .

5) Croisement en retour $ᄋ F_{1} \times \circ \cup$ basteri.

A partir des femelles hybrides $\left(F_{1}\right)$ qui, utilisées en (3), ont donné respectivement les rapports $R_{A}: 19 / 133,7 / 97,18 / 148$ et $18 / 87$, ont été effectués des croisements en retour avec des mâles basteri $(\mathrm{M})$. Ces $\mathrm{CRF}_{2}$ ont abouti à des rapports $R_{A}: 0 / 1220 / 241 / 1470 / 88$ qui diffèrent significativement de ceux du $C R F_{2}$ inverse ; le $t_{i}$, très faible, est du même ordre que celui qui caractérise la population marseillaise utilisée.

6) Défaut d'expressivité du phénotype albafusca. $\left[\left(F_{1}: \subsetneq A_{1}\right.\right.$ tricuspidata (LR) $\times$ o* U basteri (M)].

Dans les $F_{1}$ issues du croisement inverse de (2), intervient parfois une diminution de l'expressivité du phénotype albafusca : les taches blanches latérales, qui permettent de reconnaître ce phénotype, s'effacent plus ou moins complètement jusqu'à rendre très difficile sa distinction vis-à-vis du phénotype uniformis. De ce fait on observe un excès d'individus uniformis par rapport à la proportion 1/1 entre individus $A$ et $U$ prévue par l'équation (a). 
6.1) Au départ, $6 F_{1}$ de ce type ont été constituées. Les rapports $R$ relatifs au phénotype $A$ (reconnaissable comme tel), et la sex-ratio (s.r.) des $U$ sont donnés dans le tableau 1 , avec $N$ : nombre total de descendants, $n$ : nombre théorique d'individus de chaque phénotype, ainsi que la valeur du $\chi^{2}$ vis-à-vis d'une distribution $\mathrm{A} / \mathrm{U}=1$ (pour $1 \mathrm{~d}$.l.).

Dans ces 6 croisements, les femelles hybrides offrent un phénotype A typique ; il en est de même des néo-mâles $A$ issus des croisements 1,5 et 6 . Par contre, dans les descendances des croisements 2,3 et 4 , l'expressivité du phénotype $A$ est plus ou moins atténuée et c'est précisément dans ces trois croisements que le test du $\chi^{2}$ révèle un excès significatif d'individus uniformis, en quasi-totalité des mâles, dont une partie doit donc correspondre à des néo-mâles. L'équation (a) prévoit un rapport $A / U=1$; la probabilité $p$ du phénotype $U$ et celle du phénotype $A(q=1-p)$ doivent donc être égales à $1 / 2$, aux fluctuations statistiques près. $\mathrm{N}$ étant le nombre total de descendants, le nombre théorique de mâles génétiques serait donc $N p=N / 2$. L'intervalle de confiance au niveau 0,05 peut être estimé par la formule :

$$
\mathrm{Np} \pm 1,96 \mathrm{~s} \text {, où s (écart type) est égal à } \sqrt{ } \mathrm{Npq}=\sqrt{ } \frac{\mathrm{N}}{4} \text {. }
$$

Pour obtenir l'estimation des nombres maximal et minimal de néo-mâles $U$, il suffit de retrancher les valeurs limites obtenues du nombre d'individus $U$ observé. Le total des néo-mâles est évidemment égal à la somme : néo-mâles $\mathrm{A}+$ néo-mâles $U$, ce qui permet de calculer le $t_{i}$.

Les $t_{i}$ ainsi calculés, avec les intervalles de confiance, sont donnés dans le tableau 1.

6.2) A partir de femelles $A$ issues du croisement $n^{\circ} 1$ (n'ayant pas conduit à un défaut d'expressivité du phénotype $A$ ), ont été effectués 10 croisements en retour

\section{TABLEAU 1}

$\mathrm{F}_{1}: \uparrow \mathrm{A}_{1}$ tricuspidata $(\mathrm{LR}) \times \circlearrowleft \mathrm{U}$ basteri $(\mathrm{M})$

\begin{tabular}{ccccccc}
\hline $\begin{array}{c}\text { Croisements } \\
\text { et lignées } \\
\text { des o* basteri }\end{array}$ & $\begin{array}{c}1 \\
\left(\mathrm{~A}_{1}\right)\end{array}$ & $\begin{array}{c}2 \\
\left(\mathrm{~A}_{1}\right)\end{array}$ & $\begin{array}{c}3 \\
\left(\mathrm{~A}_{8}\right)\end{array}$ & $\begin{array}{c}4 \\
\left(\mathrm{~A}_{3}\right)\end{array}$ & $\begin{array}{c}5 \\
\left(\mathrm{~A}_{3}\right)\end{array}$ & $\begin{array}{c}6 \\
\left(\mathrm{~A}_{2}\right)\end{array}$ \\
\hline $\begin{array}{c}\mathrm{R}_{\mathrm{A}} \\
\text { s.r.u }\end{array}$ & $\begin{array}{l}89 / 86 \\
185 / 0\end{array}$ & $\begin{array}{c}14 / 51 \\
129 / 0\end{array}$ & $\begin{array}{c}2 / 84 \\
116 / 0\end{array}$ & $\begin{array}{c}12 / 65 \\
108 / 2\end{array}$ & $\begin{array}{c}1 / 24 \\
39 / 0\end{array}$ & $\begin{array}{c}4 / 18 \\
19 / 0\end{array}$ \\
\hline $\mathrm{N}$ & 360 & 194 & 202 & 187 & 64 & 41 \\
\hline $\mathrm{n}$ & 180 & 97 & 101 & 93,5 & 32 & 20,5 \\
\hline $\begin{array}{c}\mathrm{x}^{2} \text { versus } \\
\mathrm{A} / \mathrm{U}=1\end{array}$ & 0,26 & $21,10^{*}$ & $4,44^{*}$ & $5,82^{*}$ & 3,06 & 0,20 \\
\hline $\mathrm{t}_{\mathrm{i}}$ & 50,8 & $46,3 \pm 7,5$ & $15,2 \pm 11,6$ & $29,1 \pm 10,1$ & 4 & 18,1 \\
\hline
\end{tabular}

(légende dans le texte). ${ }^{*}: \chi^{2}$ significatifs 
avec des mâles basteri $\cup(M)$, dont 5 issus d'une lignée $\left(B L_{1}\right)$ et les 5 autres, issus de la lignée $A_{5}$. Aucun néo-mâle $n^{\prime}$ est apparu dans ces lignées. Les résultats ont été les suivants :

a) avec les $\circ \mathrm{U} \mathrm{BL}_{1}$ :

$\begin{array}{lrrrrr}\text { R A } & 0 / 7 & 1 / 9 & 4 / 147 & 2 / 88 & 1 / 118 \\ \text { s.r.u } & 10 / 0 & 7 / 0 & 121 / 0 & 87 / 0 & 117 / 0\end{array}$

Les rapports $A / U$ ne diffèrent significativement de $1 / 1$ dans aucun de ces croisements et les individus $A$ ont un aspect typique. Les $t_{i}$ sont très faibles $10 \mathrm{p}$. $100 ; 10$ p. $100 ; 2,6$ p. $100 ; 2,2$ p. $100 ; 0,8$ p. 100$)$.

b) avec les or $\cup A_{5}$ :

$\begin{array}{lrrlrr}\text { R A } & 9 / 98 & 20 / 69 & 24 / 23 & 16 / 94 & 30 / 86 \\ \text { s.r.u } & 191 / 1 & 141 / 0 & 96 / 1 & 207 / 2 & 130 / 0\end{array}$

On retrouve dans les 5 croisements une baisse d'expressivité du phénotype $A$, avec un excès d'individus $U$, qui n'est significatif que dans les 4 premiers croisements.

Les $t_{i}$, calculés à l'aide de la méthode précédemment exposée, sont respectivement pour les 4 premiers croisements :

$$
33,5 \pm 7,5 \text { p. } 10038,9 \pm 7,8 \text { p. } 100 \quad 67,1 \pm 5,3 \text { p. } 100 \quad 40,3 \pm 6,5 \text { p. } 100 .
$$

Le $t_{i}$ du croisement (5) où l'expressivité du phénotype $A$ chez les néo-mâles est réduite (mais l'excès de mâles $U$ non significatif) est de $28,9 \pm 8,8$ p. 100. Il a été vérifié que les rares femelles $U$ apparues en 6.1 et 6.2 b sont bien des néofemelles (ZZ) : croisées avec un mâle $U Z Z$, elles n'ont donné que des individus $U$, tous mâles.

\section{7) Discussion.}

7a) - En désignant par les indices LR et $M$ les génotypes respectivement rochellais et marseillais, la constitution $W Z+2 a$ (autosomes) des $\subsetneq \subsetneq$ hybrides et des néo- $O^{*} O^{*}$ hybrides de phénotype $A$ issus de la $F_{1}\left(I_{2}\right)$ peut être représentée par la formule $W_{M} Z_{L R}+a_{L R}+a_{M}$. Dans les croisements en retour successifs effectués avec des $\sigma^{*} O^{*}$ tricuspidata, les néo-mâles $A$ et les femelles $A$ obtenus conservent le segment hétérologue du chromosome $W$ de la génitrice initiale marseillaise, porteur du gène $A$, tandis que le segment homologue de ce chromosome peut s'enrichir en gènes rochellais par crossing-over avec le chromosome $Z$ qui est toujours purement du type rochellais; la constitution hétérosomique est donc: $W_{M / L R} Z_{L R}$; les autosomes comportent pour moitié une garniture rochellaise et pour l'autre moitié une garniture hybride où le dosage $M / L R$ est de plus en plus en faveur des autosomes rochellais.

Cette étude n'est que préliminaire. La grande variabilité du taux d'inversion observée en $F_{1}$ et en $\mathrm{CRF}_{2}$ ouvrait la possibilité de sélections quantitatives vers le haut et vers le bas. Partant d'un $\mathrm{CRF}_{2}$ de rang élevé (18/87), non significativement différent du maximum observé $(28 / 98)\left(\chi^{2}=0,63\right)$, il a été choisi, dans ce premier temps, une sélection vers le haut en $\mathrm{CRF}_{3}$. Les résultats obtenus constituent, avec le $R_{A}$ du $C R F_{2}$ d'origine, un ensemble homogène $\left(\chi^{2}=6,12-\right.$ d.I. $\left.=4\right)$, le $t_{i}$ moyen des $\mathrm{CRF}_{3}$ étant identique (18 p. 100) à celui de la descendance $\mathrm{CRF}_{2}$. 
Partant alors de l'une des descendances $\mathrm{CRF}_{3}$ (celle qui comportait le plus faible $\mathrm{t}_{\mathrm{i}}$ mais ne différait pas significativement des trois autres), les $2 \mathrm{CRF}_{4}$ obtenus, homogènes entre eux, constituent, avec le $\mathrm{CRF}_{3}$ de départ, un ensemble hétérogène $\left(\chi^{2}=15,38-d . I\right.$. $\left.=2\right)$; le $t_{\mathrm{i}}$ moyen des $\mathrm{CRF}_{4}$ étant plus élevé que celui du départ $(34,9$ p. 100), ceci montrait que la sélection vers le haut restait possible. En fait, les $2 \mathrm{CRF}_{5}$ effectués (homogènes entre eux) ont constitué avec le $\mathrm{CRF}_{4}$ de départ $\left(R_{A}=26 / 53\right)$ un ensemble homogène et le $t_{i}$ obtenu (28 p. 100) n'a donc pas progressé : il s'est en fait stabilisé au niveau de celui de la lignée rochellaise $A_{1}$. Un développement de lignées à partir de descendances à taux nul de néomâles sera abordé dans une prochaine publication.

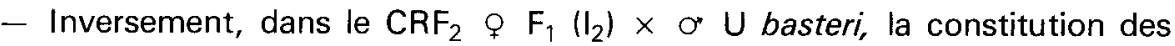
néo-mâles $A$ et des femelles $A$ obtenus peut être représentée par la formule : $W_{M / L R} Z_{M}+a_{M}+a_{L R / M}$. Le renforcement du génotype marseillais, à la fois au niveau de $Z$ et des autosomes, peut être considéré comme responsable du faible taux de néo-mâles $A$, en dépit des crossing-over susceptibles d'être intervenus au niveau du segment homologue de $W$ lors de la gamétogenèse des femelles $F_{1}$ et ayant apporté des gènes rochellais.

Ainsi, la divergence des résultats observée entre les 2 types de $C R F_{2}$, d'une part, l'accroissement du taux des néo-mâles constaté au cours des croisements en retour successifs avec des mâles rochellais, d'autre part, conduisent à la conception que le déterminisme de l'inversion du sexe fait intervenir des gènes multiples, autosomiques et peut-être également partiellement liés au sexe. L'intervention de gènes multiples est déjà sensible dans le $C R F_{2}: \subsetneq F_{1} \times \sigma^{\prime} L R$. En effet, les taux de néo-mâles dans les 11 croisements constituent une variable continue, ce dont peut rendre compte un dosage variable de gènes, les uns favorables, les autres défavorables à l'inversion, lors de la gamétogenèse des femelles de la $F_{1}$. Une poursuite de la tentative de sélection pourrait nous renseigner sur le nombre de loci impliqués dans le déterminisme de l'inversion du sexe femelle.

II est à remarquer que ces gènes multiples ont beaucoup plus de raison d'être essentiellement autosomiques que partiellement liés au sexe car, si l'enrichissement de la constitution chromosomique hybride en autosomes rochellais est assuré à chaque croisement en retour, les crossing-over entre un segment du $Z$ rochellais, porteur de gènes favorables, et $W$ ont beaucoup moins de chance de se réaliser.

7b) Dans la plupart des croisements réalisés entre femelle $A$ et mâle $U$, le rapport $A / U$ ne diffère pas significativement de 1 ; la pénétrance du gène $A$ est complète, y compris chez les néo-mâles. Par contre en (6), dans les croisements entre femelles A tricuspidata et certains mâles $U$ basteri, s'observe une baisse de l'expression du gène $A$, le phénotype devenant, à l'extrême, indiscernable de $\mathrm{U}$, d'où un rapport $A / U<1$, et ce phénomène ne s'observe que chez les néo-mâles. Le taux des inversés sexuels, englobant l'éventail des néo-mâles dont le phénotype va de $A$ à $U$, est alors le plus souvent supérieur à celui observé en cas de pénétrance complète du gène $A$. Contrairement aux résultats du paragraphe 5 , l'enrichissement du génotype hybride en gènes basteri ne s'accompagne pas d'une chute du taux d'inversion sexuelle des femelles génétiques. Effectivement, 
la comparaison, à l'aide $d u \chi^{2}$, de l'ensemble des rapports $R_{A}$ obtenus en $F_{1}$ et en $\mathrm{CRF}_{2}$, dans les descendances offrant une baisse d'expressivité du phénotype $A$ (et en même temps un excès significatif de U) montre qu'il s'agit d'un ensemble homogène $\left(\chi^{2}=7,62\right.$ pour 6 d.I.). L'enseignement qui se dégage de ces résultats est le fait d'une corrélation étroite entre, d'une part, l'inversion sexuelle des femelles hybrides : $W_{A(L R)} Z_{M} a_{L R} a_{M}$ en $F_{1}, W_{L R / M} Z_{M} a_{M} a_{L R / M}$ en $C_{R} F_{2}$ (que l'on peut traduire comme une inhibition du déterminant sexuel femelle majeur situé sur le segment différentiel de l'hétérochromosome W) et d'autre part, un processus du même ordre atteignant le gène qui contrôle la coloration albafusca. Ceci suggère que ce dernier pourrait être situé sur le segment d'ADN contrôlant le sexe femelle. Cette hypothèse rejoint celle qui a été formulée par Tinturier-Hamelin (1963), et celle de Winge et Ditlevsen (1948), concernant également des gènes de coloration, chez les Téléostéens Cyprinodontiformes.

\section{II. - Croisements faisant intervenir d'autres phénotypes colorés}

Parallèlement à l'utilisation de femelles basteri de phénotype $A$, a été testée l'aptitude des femelles de la même sous-espèce, mais de phénotype bilineata ou uniformis, à offrir des néo-mâles dans leur descendance lorsqu'elles sont croisées avec un mâle tricuspidata $U$ de La Rochelle.

1) Phénotype bilineata (B).

Dans les lignées utilisées, le gène $B$, bien que situé sur le segment pair de $W$, est strictement lié à ce chromosome par suite de la présence d'un inhibiteur de crossing-over (Tinturier-Hamelin, 1963).

1a) Croisement $Q$ B (basteri) $\times$ or U (tricuspidata).

Ont été employées 5 femelles basteri $\mathrm{B}$, dont 3 récoltées à Marseille (Pointe Rouge), une au Racou et une à Tunis.

Rappelons que le phénotype $B$ est limité au sexe femelle et que les mâles sont donc uniformis (Tinturier-Hamelin, 1963). De ce fait, si une inversion sexuelle des filles se manifeste lors du croisement $\& \mathrm{~B} \times \mathrm{O}^{\mathrm{U}} \mathrm{U}$ tricuspidata, elle se marquera par une réduction du nombre des femelles $B$ vis-à-vis de celui des mâles $U$. Seule, sur les 5 femelles utilisées, la femelle B de Tunis - qui, croisée au préalable avec un mâle basteri $U$ de la même population avait donné une sex-ratio normale ( 43 q \& B contre 48 ơ ơ U) - a offert, croisée avec un mâle tricuspidata de La Rochelle, une forte arrhénogénie : 6 \% $\mathrm{B}$ contre 152 or o $^{\mathrm{U}} \mathrm{U}$, ce qui peut être compris comme résultant d'une inversion sexuelle atteignant environ 92 p. 100 des femelles. Pour montrer que le phénotype $U$ des mâles de cette $F_{1}$ correspondait bien à deux génotypes : $W_{B} Z$ (néo-mâles) et $Z Z$ (mâles) en proportions presque égales, $18 \mathrm{CRF}_{2}$ ont été effectuées entre ces mâles $U$ et des femelles basteri $U$ de Marseille. Lorsqu'il s'agit de néo-mâles, ces croisements doivent faire apparaître des individus bilineata. C'est ce qui s'est réalisé dans 10 croisements.

On observe ainsi pour le phénotype $B$ des résultats qui paraissent voisins de ceux qui concernent le phénotype $A$, à savoir que seules certaines femelles $B$ sont favorables à l'inversion de leurs filles. Lorsque la femelle $B$ est favorable à l'inver- 
sion, le taux des néo-mâles est alors bien plus fort que celui observé dans les croisements du même type, effectués avec des femelles $A$.

1b) Croisement : néo- $\sigma$ A (tricuspidata) $\times$ \& (basteri).

Les néo-mâles utilisés (provenant de la lignée $A_{1}$ de La Rochelle) offraient tous un phénotype $A$ net. Les femelles basteri $B$ proviennent de la deuxième génération de femelles des lignées $B_{1}$ et $B_{5}$ de Marseille qui n'ont donné aucun néo-mâle.

Un tel croisement répond à l'équation hétérosomique :

b $W_{A} Z$ (néo- $\left.0^{*}\right) \times W_{B} Z \rightarrow W_{A} Z+W_{B} Z+W_{A} W_{B}+Z Z$ qui prévoit une égalité numérique entre les individus des 4 phénotypes: $A, B, A B$ et $U$ et une sexratio 1 or/3 q 9 .

Sur 8 croisements réalisés, 5 ont été féconds. Les rapports $\mathrm{R}$ correspondants aux 3 phénotypes colorés et la sex-ratio (s.r.) des individus $U$ sont relatés dans le tableau 2 qui indique, en outre, le nombre total des descendants (N), le nombre théorique d'individus par phénotype $(N / 4=n)$, le $\chi_{2}$ versus une distribution $A / U=1$, enfin les $t_{i A}$ avec leurs intervalles de confiance. II apparaît que dans les 5 croisements les nombres d'individus obtenus concernant les phénotypes $B$ et $A B$ ne diffèrent pas significativement de ceux prévus par l'équation (b) ; il n'est apparu aucun néo-mâle, ce qui rappelle les résultats des croisements (II.1a) entre des femelles B de la même lignée basteri et des mâles tricuspidata U. En ce qui concerne les proportions d'individus de phénotypes $A$ et $U$, seul le résultat du croisement (4) $(24 / 28)$ ne diffère pas significativement de la prévision. Dans les quatre autres croisements, le nombre de mâles $U$ est significativement plus élevé que celui des individus $A$, et il a été constaté une baisse d'expressivité du phénotype albafusca, uniquement chez les néo-mâles $A$, en (1) et (3). Du fait qu'en (2) et (5), le nombre de $U$ ne diffère pas significativement du nombre théorique, il se peut que la faible représentation des $A$ soit due à une mortalité différentielle

TABLEAU 2

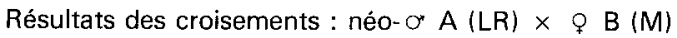

\begin{tabular}{cccccc} 
Croisements & 1 & 2 & 3 & 4 & 5 \\
\hline $\mathrm{R}_{\mathrm{A}}$ & $4 / 25$ & $4 / 5$ & $11 / 8$ & $1 / 23$ & $0 / 8$ \\
$\mathrm{R}_{\mathrm{B}}$ & $0 / 49$ & $0 / 21$ & $0 / 46$ & $0 / 20$ & $0 / 25$ \\
$\mathrm{R}_{\mathrm{AB}}$ & $0 / 49$ & $0 / 22$ & $0 / 37$ & $0 / 32$ & $0 / 18$ \\
$\mathrm{~s} . \mathrm{r} \cdot \mathrm{U}$ & $75 / 0$ & $23 / 0$ & $59 / 0$ & $28 / 0$ & $22 / 0$ \\
\hline $\mathrm{N}$ & 202 & 75 & 161 & 104 & 73 \\
\hline $\mathrm{n}$ & 50,5 & 18,75 & 40,35 & 26 & 18,25 \\
\hline$\chi^{2}$ vs A/U =1 & $21,03^{*}$ & $6,02^{*}$ & $19,86^{*}$ & 0,30 & $6,52^{*}$ \\
\hline (d.I. $=1)$ & $50 \pm 9,6$ & $(44)$ & $78,4 \pm 4,8$ & 4,1 & $(0)$ \\
\hline $\mathrm{t}_{\mathrm{iA}}$ & & & & & \\
\hline
\end{tabular}


intervenue avant la naissance. II n'a pas été tenu compte de ces croisements. Les $t_{i} A$ (voir tableau) ont été calculés comme précédemment.

Ces résultats apportent donc une confirmation de ceux qui ont été exposés en I.6.

2) Phénotype uniformis.

Ont été utilisées 7 femelles basteri $U$, dont 4 récoltées à Tunis, 2 à Marseille et 1 au Racou. Croisées avec des mâles basteri de ces populations, elles ont donné des sex-ratio normales.

2a) Croisement : $q U$ (basteri) $\times \circ U$ (tricuspidata).

Croisées avec des mâles tricuspidata $U$ de La Rochelle, seules trois femelles, à savoir les 2 femelles de la population de Marseille et une des femelles de Tunis, ont donné des descendances arrhénogènes: $24 \sigma^{\prime} \sigma^{*}, 3 q \%-890^{\prime \prime} \sigma^{\prime}$, 27 ๆ-206 Ơ $\sigma^{*}, 4$ \% , ce qui peut s'interpréter comme résultant d'une inversion sexuelle des filles variant, dans le croisement (1), entre $74,09 \pm 9,77$ p. 100 ; en (2) : $51,85 \pm 8,76$ p. 100 ; en (3): $96,11 \pm 0,56$ p. 100 . Le phénotype uniformis permet donc de retrouver des résultats comparables à ceux observés avec le phénotype $B$, à savoir que certaines femelles basteri offrant ce phénotype sont très favorables à l'inversion sexuelle de leurs filles lorsqu'elles sont croisées avec un mâle tricuspidata $U$ rochellais.

2b) Croisement : néo- ${ }^{*} A$ (tricuspidata) $\times \& \cup$ (basteri) (M).

Ont été utilisés les néo-mâles ayant fécondé les femelles $B$ (en II.1b) lors des croisements $1,2,3$ et 5 . Un tel croisement répond à l'équation hétérosomique

c $\quad W_{A} Z \times W Z \rightarrow W_{A} Z+W_{A} W+W Z+Z Z$, qui prévoit une égalité numérique entre individus $A$ (qui sont tous des femelles génétiques) et $U$, chez lesquels la sex-ratio est de 1.

TABLEAU 3

Résultats des croisements : néo- $\sigma^{*} A(L R) \times \odot U(M)$

\begin{tabular}{ccccc}
\hline Croisements & 1 & 2 & 3 & 5 \\
\hline $\mathrm{R}_{\mathrm{A}}$ & $2 / 35$ & $4 / 28$ & $3 / 23$ & $1 / 29$ \\
\hline $\mathrm{n}_{\mathrm{A}}$ & $0 / 32$ & $0 / 43,5$ & $0 / 26,5$ & $0 / 37,5$ \\
\hline s.r. réelle $(\mathrm{U})$ & $21 / 6$ & $45 / 10$ & $18 / 9$ & $45 / 0$ \\
\hline s.r. théorique $(\mathrm{U})$ & $16 / 16$ & $21,75 / 21,75$ & $13,25 / 13,25$ & $18,75 / 18,75$ \\
\hline$\chi^{2}$ vs $\mathrm{A} / \mathrm{U}=1$ & 1,56 & $6,08^{*}$ & 0 & 3 \\
\hline$\chi^{2}$ vs s.r. $(\mathrm{U})=1$ & $7,81^{*}$ & $31,19^{*}$ & 3,06 & $55,48^{*}$ \\
\hline $\mathrm{t}_{\mathrm{i}} \mathrm{A}$ & 5,4 & $32,6 \pm 14,1$ & 11,5 & 3,3 \\
\hline $\mathrm{t}_{\mathrm{i}} \mathrm{U}$ & $18,5 \pm 15,6$ & $41,3 \pm 28,7$ & $9,4 \pm 9,4$ & 100 \\
\hline
\end{tabular}

(légende dans le texte). ${ }^{*}: \chi^{2}$ significatifs 
Les rapports $R$ obtenus dans chaque phénotype sont indiqués dans le tableau 3, avec les nombres théoriques d'individus $A\left(n_{A}\right)$, les sex-ratio réelle et théorique des $U$, les $\chi^{2}$ versus la distribution $A / U=1$, et versus une sex-ratio des $U$ égale à 1 (pour 1 d.I.), enfin les $t_{i} A$ et les $t_{i} U$, calculés comme précédemment. II apparaît que dans les croisements $n^{0 s} 1,3$ et 5 le nombre des $A$ est non significativement différent de celui que prévoit l'équation (c) et les néo-mâles offrent un phénotype A net. II y a eu, par contre, inversion sexuelle d'un certain nombre de femelles $U$ dans le croisement $n^{\circ} 1$. Dans le croisement $n^{\circ} 3$, la sex-ratio des $U$ ne diffère pas significativement de 1 ; par contre, dans le croisement $n^{\circ} 5$, il y a eu inversion de la totalité des femelles $U$ en néo-mâles : un croisement ultérieur de la génitrice $U$ avec un mâle basteri (lignée $U_{1}$ ) a en effet prouvé qu'il s'agissait bien d'une femelle génétique : la descendance a été de $43 \sigma^{\circ} 0^{\prime}, 44 \%$ \% . Dans le croisement $n^{\circ} 2$, le rapport $A / U$ diffère significativement de 1 . On peut donc affirmer que figurent, parmi les mâles uniformis, des individus porteurs du gène $A$.

3) Discussion.

La comparaison des résultats obtenus en (1b) et (2b) en croisant le même néo-mâle tricuspidata $A$ avec deux femelles basteri, respectivement $B$ et $U$, ne peut porter utilement que sur les croisements $n^{\text {os }} 1$ et 3 , en raison de la déficience anormale des $A$ dans les croisements $(1 b) n^{\text {os }} 2$ et 5.

Dans les deux croisements nos 1 et 3 : avec les femelles $B$, il y a eu baisse de l'expressivité du phénotype $A$, accompagnée de forts $t_{i}$ (centrés respectivement autour de 50 et 70 p. 100) ; avec les femelles U, l'expressivité de A a été complète et les $t_{i} A$ faibles (respectivement 5,4 p. 100 et 11,5 p. 100). En fait, comme nous le verrons dans un autre travail, il est possible qu'en (2b) l'inversion n'intervienne que chez les femelles $W Z$ (et non les $W W$ ), ce qui porterait les $t_{i} A$, chez les premières, respectivement à $12 \pm 3,8$ et $28,3 \pm 11,1$, valeurs restant nettement plus faibles que celles qui accompagnent la baisse d'expressivité de $A$.

La comparaison des taux d'inversion concernant, dans les 4 croisements de (2b), les femelles $A$ et $U$, montre des différences susceptibles d'être considérables [exemple dans le croisement $n^{\circ} 5$, où le $t_{i} A$ est globalement de 3 p. 100 lou $7,5 \pm 2,8$ p. 100 s'il ne porte que sur les femelles $W Z$ ) alors qu'il est de 100 p. 100 chez les femelles U]. S'agissant de femelles sceurs, qui ne diffèrent, en principe, que par la structure de I'hétérochromosome $W\left(W_{A}\right.$ et $\left.W_{A}+\right)$, on peut penser que la faculté d'inversion dépend, non seulement du complexe autosomique hybride, mais également de la structure de $W$.

\section{Discussion générale et conclusion}

Les Crustacés répondent au schéma général de la détermination du sexe fondé sur I'homo-hétérogamétie. Lorsque les preuves reposant sur l'examen du caryotype manquent - ce qui est souvent le cas (et notamment chez Idotea balthica) - la constitution hétérosomique a été révélée par l'étude de l'hérédité liée au sexe et (ou) par l'emploi de néo-mâles expérimentaux. Ainsi, pour ne citer que les Malacostracés, le mâle est hétérogamétique chez les Décapodes, certains Isopodes et Amphipodes; c'est par contre I'inverse chez d'autres Isopodes, Idotea 
balthica par exemple (références in Bacci, 1965 ; Legrand et al., 1974 ; LegrandHamelin, 1977). L'antigène $H_{y}$ a été décelé par Shalev et al. (1980) chez le mâle de Homarus americanus, ce qui est considéré comme conforme à l'hétérogamétie mâle observée chez les Décapodes.

Du fait que la sex-ratio des descendances répond le plus souvent à l'équation hétérosomique proposée, celle-ci peut traduire l'existence d'un déterminisme génétique réel et simple du sexe, par exemple, du type $\mathrm{Fm}(q) \times \mathrm{mm}\left(\mathrm{O}^{\circ}\right)$, applicable à la plupart des croisements intervenant dans les populations d'/dotea basteri. La liaison stricte du sexe femelle (génétique, mais non phénotypique) avec le phénotype albafusca, tend à confirmer l'existence, sur le segment différentiel de $W$, d'un déterminant femelle majeur. Cependant, une telle formulation de la détermination du sexe est en défaut lorsqu'apparaissent des inversés sexuels. Elle est encore plus en défaut lorsqu'il s'agit d'expliquer les phénomènes de monogénie, très fréquents chez les Crustacés Isopodes terrestres (références in Legrand, 1977).

Si de nombreux auteurs ont invoqué l'intervention de gènes autosomiques dans la réalisation du phénotype sexuel, ceci est resté théorique dans bien des cas. Chez les Oniscoïdes, De Lattin (1958) accorde à des gènes autosomiques contrôlant la coloration du corps des rôles de réalisateurs du sexe, qui agiraient à l'intérieur d'un système polyfactoriel, en l'absence (pensait-il) d'hétérochromosomes. Il est connu, chez la Drosophile - où la théorie de Bridges (1914) de la balance sexuelle est toujours actuellement considérée comme la meilleure explication de la détermination du sexe (McCarrey et Abbott, 1979) - que des gènes autosomiques ont une action importante sur le sexe (références in Baker et Ridge, 1979). Le rôle de déterminants sexuels a été dévolu à des gènes multiples autosomiques chez les Téléostéens Cyprinodontiformes, à la suite des travaux de Winge et Ditlevsen (1948), dont les résultats ont été étendus par d'autres auteurs (références in Jalabert et al., 1971 et Hammerman et Avtalion, 1979).

Les résultats concernant /dotea balthica exposés ici peuvent être résumés ainsi : a) En premier lieu, il a été constaté (en 1.3 et 4) que l'enrichissement progressif du génotype des femelles hybrides basteri-tricuspidata en gènes susceptibles d'être partiellement liés au sexe, mais probablement essentiellement autosomiques, de type tricuspidata, s'accompagne d'une augmentation du taux des néo-mâles, qui atteint finalement un niveau comparable à celui de la lignée rochellaise à l'origine des femelles albafusca utilisées. La réciproque a été observée (en I.5) par enrichissement du génotype hybride en gènes basteri : le taux des inversés est ramené au niveau, très faible, qui caractérise la sous-espèce basteri.

Ces résultats tendent à montrer que l'inversion du sexe femelle est sous le contrôle de plusieurs loci occupés, chez la plupart des individus de la sous-espèce basteri, surtout par des allèles homozygotes défavorables à l'inversion et dominants par rapport aux gènes homologues tricuspidata favorables à l'inversion. Quelle que soit cependant l'importance de l'effet de ces gènes chez tricuspidata (et chez les hybrides issus de croisement en retour multiples entre les deux sousespèces), le taux des néo-mâles ne dépasse pas la valeur limite observée chez les individus de phénotype $A$ de la lignée $A_{1}$ (LR). Il existerait donc, chez beaucoup de ces derniers, une "parade " à cette inversion du sexe, qui porterait sur le 
niveau où agissent ces gènes multiples, cest-à-dire le ou les déterminant(s) femelle(s) majeur(s). L'inversion sexuelle des femelles tricuspidata intervenant, non seulement au laboratoire, mais également dans la nature, on peut penser que sa limitation est protégée par la sélection naturelle. Il est à souligner que cette limitation du taux d'inversion n'intervient pas, ou peu, lorsque certaines femelles basteri $\mathrm{B}$ ou $\mathrm{U}$ sont croisées avec des mâles tricuspidata, puisque le taux d'inversion des femelles peut atteindre 100 p. 100 (cf. II.1a et 2a). Mais il s'agit là de croisements qui n'intervienennt qu'au laboratoire (sauf peut-être au niveau du détroit de Gibraltar). b) En second lieu, le croisement entre des femelles tricuspidata $A(L R)$ et des mâles basteri issus de certaines lignées marseillaises affaiblit l'expressivité du phénotype $A$ jusqu'à le rendre indiscernable du type sauvage, $U$. Ce phénomène s'accompagne toujours de l'inversion du sexe des femelles hybrides, quel que soit le degré de réduction de l'expressivité, et, le plus souvent, d'une hausse importante de ce taux d'inversion (cf. Il.1b et 2b).

Quelle interprétation peut-on donner de ces résultats?

Ce point $b$ apporte une confirmation du rôle de $W$ comme porteur d'un déterminant femelle majeur étroitement lié au gène $A$. Le complexe génique hybride responsable à la fois de la baisse de l'expressivité du phénotype $A$ et de l'inversion sexuelle des femelles doit être distinct de celui qui contrôle l'inversion sexuelle accompagnée d'une expressivité complète du phénotype $A$.

Jusqu'ici, cet effet simultané n'a été observé que lors du croisement d'une femelle, ou d'un néo-mâle tricuspidata $A$ avec certains géniteurs (mâles ou femelles) basteri, et non dans le croisement inverse. Par ailleurs, la comparaison des rapports $R$ entre individus $A$ obtenus en $F_{1}$ et en $C R F_{2}$ (I.6.1 et I.6.2b), montre que l'enrichissement en gènes basteri du génotype hybride, contrairement à ce qui a été observé en (1.5) dans le cas d'un maintien de la pénétrance complète du gène $A$, ne modifie pas le taux d'inversion des femelles. Ces données amènent à penser que l'effet simultané sur le phénotype $A$ et le phénotype sexuel des femelles génétiques pourrait dépendre, d'une part, de la structure du $W$ des femelles tricuspidata, d'autre part, d'un petit nombre de gènes, autosomiques ou liés au chromosome $Z$ des géniteurs basteri, agissant - comme le complexe génique invoqué en (a) - en inhibiteurs du déterminant sexuel majeur porté par $W$. Dans cette interprétation, contrairement à celle de Winge, les gènes autosomiques, ou partiellement liés au sexe, invoqués en (a) et (b) ne sont pas considérés comme de véritables déterminants sexuels mâles mais comme des modificateurs c'est-àdire influençant indirectement la réalisation du phénotype sexuel, tel qu'il en intervient dans celle de multiples caractères somatiques. II faut souligner que des modificateurs du même type, mais dont l'effet est d'inverser les mâles génétiques $\mathrm{ZZ}$ en néo-femelles (cf. 1.6.2b), existent également (donc hors de l'hétérochromosome W), comme l'a déjà signalé l'un de nous (Tinturier-Hamelin, 1963).

La détermination du sexe doit ainsi être considérée comme polyfactorielle puisque, quels que soient le nombre et la position des gènes sexuels majeurs, ils constituent, avec les deux types de modificateurs précités, un complexe génique dont dépend le phénotype sexuel. L'effet de ces croisements entre sous-espèces d'/dotea balthica est comparable à celui que Goldschmidt (1955) a observé dans les croisements entre " races » de Lymantria dispar, à ces différences près que, 
chez /dotea, l'inversion du sexe, d'une part, intervient spontanément dans les populations naturelles, d'autre part, est complète, sans apparition d'intersexués. Ces phénomènes d'inversion spontanée (ou provoquée au laboratoire par croisement de sous-espèces) témoignent d'un stade primitif de la détermination du sexe chez les Crustacés Isopodes.

L'étude, dans une prochaine publication, de l'inversion du sexe des femelles de phénotype bilineata-lineata apportera des données complémentaires sur le mécanisme de la détermination du sexe chez ldotea balthica.

Reçu en décembre 1981. Accepté en mars 1982.

\section{Références}

ARTAULT J. C., 1977. Contribution à l'étude des garnitures chromosomiques chez quelques Crustacés /sopodes. Th. $3^{\mathrm{e}}$ cycle, Poitiers, $\mathrm{n}^{\circ} 665$.

BACCI G., 1965. Sex determination. Pergamon Press.

BAKER B. S., RIDGE K. A., 1979. Sex and the single cell. I. On the action of major loci affecting sex determination in Drosophila melanogaster. Genetics, 94, 383-423.

BRIDGES C. B., 1914. Direct proof through nondisjonction that the sex-linked genes are borne on the X-chromosome. Science, 40, 107-109.

GOLDSCHMIDT R.B., 1955. Theoretical genetics. Univ. California Press, Berkeley, Los Angeles.

HAMMERMAN I. S., AVTALION R. R., 1979. Sex determination in Sarotherodon (Tilapia). II : The sex-ratio as a tool for the determination of genotype. A model of Autosomal and Gonosomal influence. Theor. appl. Genet, 55, 177-187.

JALABERT B., KAMMACHER P., LESSENT P., 1971. Déterminisme du sexe chez les hybrides entre Tilapia macrochir et Tilapia nilotica. Etude de la sex-ratio dans les recroisements des hybrides de première génération par les espèces parentes. Ann. Biol. anim. Bioch. Biophys., 11, 155-165.

FAVERGE J. M., 1962. Méthodes statistiques en psychologie appliquée. P.U.F., Paris.

LATTIN G. De, 1958. Vererbungsstudien an Isopoden. I. Zur Genetik der Farbvariabilität von Cylisticus convexus (De G.). Zool. Anz., 160, 313-324.

LEGRAND J. J., 1977. Détermination génétique et épigénétique du sexe chez les Crustacés Isopodes terrestres. Corrélation entre monogénie et intersexualité. Bull. Soc. Sci. nat. Tunisie, 12, 3-12.

LEgRAND J. J., JUChAUlt P., ARTAULT J. C., MOCQUARD J. P., PICAUD J. L., 1974. Le statut systématique de la « forme " petiti Vandel de Porcellio dilatatus Brandt récoltée dans I'île Saint-Honorat (Alpes-Maritimes). Critères morphologiques, génétiques et physiologiques. Bull. Soc. Zool. Fr., 99, 461-471.

LEGRAND-HAMELIN E., 1977. Obtention de néo-mâles fonctionnels et démonstration expérimentale de I'hétérogamétie femelle chez /dotea balthica (Crustacé Isopode). C. R. Soc. Biol., 171, $176-180$.

MCCARREY J. R., ABBOT U. K., 1979. Mechanisms of genetic sex determination, gonadal sex differenciation, and germ-cell development in animals. In Adv. Genetics, 20, 217-290, Acad. Press New York.

SHALEV A., GOLDENBERG P. Z., HUEBNER E., 1980. Evidence for an $\mathrm{H}-\mathrm{Y}$ crossreactive antigen in Invertebrates. Differentiation, 16, 77-80.

TINTURIER-HAMELIN E., 1963. Polychromatisme et détermination génétique du sexe chez l'espèce polytypique /dotea balthica (Pallas) (Isopode Valvifère). Cah. Biol. mar., 4, 473-591.

WINGE Ö., DITLEVSEN E., 1948. Colour inheritance and sex determination in Lebistes. C. R. Labor. Carlsberg, Ser. Physiol., 24, 227-248.

YAMAMOTO T., 1959. A further study in induction of functional sex-reversal in genotypic males of the medaka (Oryzias latipes) and progenies of sex reversal. Genetics, 44, 739-757. 